\title{
Modeling and Simulation of a Fault-Tolerant Electromechanical Actuation System for Helicopter Swashplates in Modelica
}

\author{
Sebastian Seemann \\ EADS Innovation Works \\ TCC6 Energy and Propulsion \\ 81663 Munich, Germany \\ sebastian.seemann@eads.net
}

\author{
Clemens Schlegel \\ Schlegel Simulation GmbH \\ Meichelbeckstr. 8b \\ 85356 Freising, Germany \\ cs@schlegel-simulation.de
}

\begin{abstract}
Replacing hydraulic primary flight control actuators by electromechanical actuators imposes the problem of reduced reliability. This problem may be overcome by using redundant actuators what in turn increases the system complexity. The appropriate redundancy level and component mapping must be assessed. In specific failure cases the system must be reconfigured in order to maintain the specified performance level to meet aircraft safety regulations. The assessment of the system's reaction upon such kind of scenarios is however a complicated task and must be supported by modeling and simulation. Therefore, modeling and simulation of such a faulttolerant electromechanical system in Modelica is described in this paper. Sample simulation results are presented and discussed.
\end{abstract}

Keywords: electromechanical actuator; redundancy; faultl-tolerance; over-determined kinematics; helicopter; swashplate; flight controls;

\section{Introduction}

A general trend in aviation is to replace hydraulic subsystems like primary flight control actuators by electromechanical devices. However, substituting a hydraulic actuator by an electromechanical actuator (EMA) has the disadvantage of reduced component reliability. This accompanies two major challenges. First, in order to meet aircraft safety regulations higher degrees of redundancy are needed for the utilization of EMAs. Moreover, in the case a redundant actuator jams mechanically, it must be disconnected from the swashplate to maintain controllability of the remaining actuators and the ability to position the entire swashplate.

The system under investigation is therefore specified to provide fail-operative behavior for major mechanical failures and dual-fail-operative behavior for combinations of any other failures. This requires certain degrees of redundancy of all system parts and meaningful mapping of the components in order to allow for failures while maintaining function and performance. Furthermore, suitable means for failure detection, failure isolation and system reconfiguration are needed.

\section{System architecture and compo- nent failures}

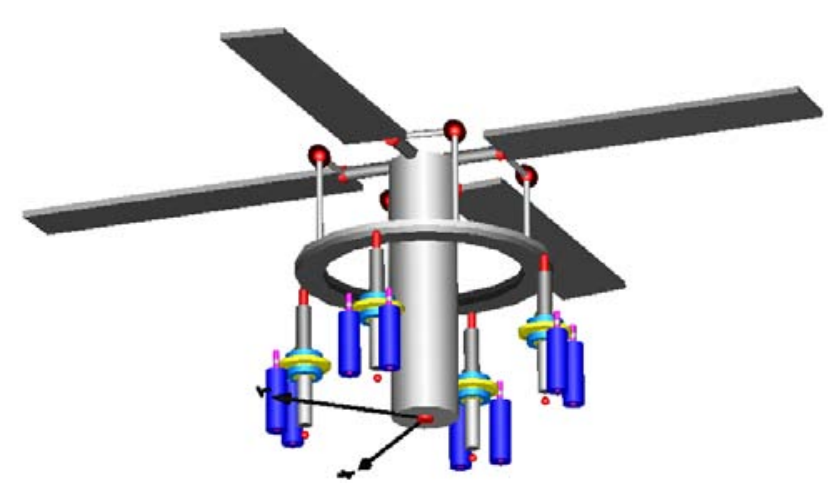

Figure 1: Swashplate actuation system

The concept investigated comprises four vertically arranged and equidistantly spaced actuators for the operation of a three degree of freedom helicopter swashplate, each of them containing two motors (see figure 1, blue cylinders). The system operates against aerodynamic forces caused at the rotor blades and exerted on the rotating upper ring of the swashplate through pitch links. The stationary lower ring of the 
swashplate is positioned by the four EMAs. All actuators are simultaneously active to achieve a minimum of nominal loading. The provided redundancy allows for the malfunction of one actuator unit, the three remaining EMAs safely continuing control of the swashplate with reduced performance.

\subsection{Swashplate actuator}

Each swashplate actuator consists of two electric motors in torque-summing configuration and a mechanical drive train. The latter comprises a two-stage gearbox, ballscrew and nut assembly, and an output piston to the swashplate attachment. The variety of conceivable mechanical failure modes can be categorized into two types of mechanical failures to be taken into account, namely fracture and jamming of the drive train.

For monitoring and control purposes each single actuator drive path is equipped with an absolute position sensor and two cut force sensors. Moreover, each of the two electric motors per actuator features sensors for angular position, phase currents, and temperature.

\subsection{Disconnect device}

Under all flight conditions, the swashplate must be controllable in three degrees of freedom, i.e. collective, pitch, and roll (see e.g. [1]). As mentioned, the risk of a mechanical jam must be considered which can be caused, for instance, by wear or debris. To avoid the swashplate getting stuck due to a single jammed actuator, fail-safe degradation of the overall actuation system is needed. For this reason, each actuator is fitted with a disconnect device, decoupling the output shaft from the mechanical drive train [2]. After disconnection of one actuator the swashplate is still safely controlled by the remaining three actuators. However, the time needed for disconnection is critical regarding stability and stress and therefore imposes strict requirements on failure detection and disconnect activation.

\section{$2.3 \quad$ Electric motor}

The most common design for electrically driven flight surface actuators is a permanent magnet synchronous motor (PMSM) fed by pulse-width modulated (PWM) inverters. This is due to the superior torque and power density of such devices.

The most common faults are device failure within the inverter and open and/ or short circuit failures in the motor windings. This failures typically lead to a loss of motor output torque (open circuit failure or inverter failure) or a drag torque induced by shortcircuit currents.

\subsection{Power supply}

The electric power sources driving the motors are also critical components of the overall system. The required power supply reliability is ensured by a multi power bus configuration. The system has four independent power supplies, each being connected to one actuator control electronics (ACE) unit. The failure cases considered include a loss of power supply output power, and out-of range output voltage.

\subsection{Redundancy and component mapping}

The maximum accepted probability of catastrophic events of an aircraft system is $1 \times 10^{-9} \mathrm{~h}^{-1}$ [3]. To meet this figure several subsystems must be redundant and the connections of subsystems must be designed such that a single failure results in minimum system degradation.

Regarding the overall drive train a static redundancy approach is followed, i.e. all actuators are simultaneously active. Each is driven by two fully independent paths of torque generation, comprising electric motors, power supply busses, power electronics, and control computers. In order to minimize system degradation after a failure the two motors of a single actuator are controlled by different ACE units. Moreover, each actuator has a different combination of motor control electronics assignment in order to avoid the loss of two entire actuators after two ACE failures.

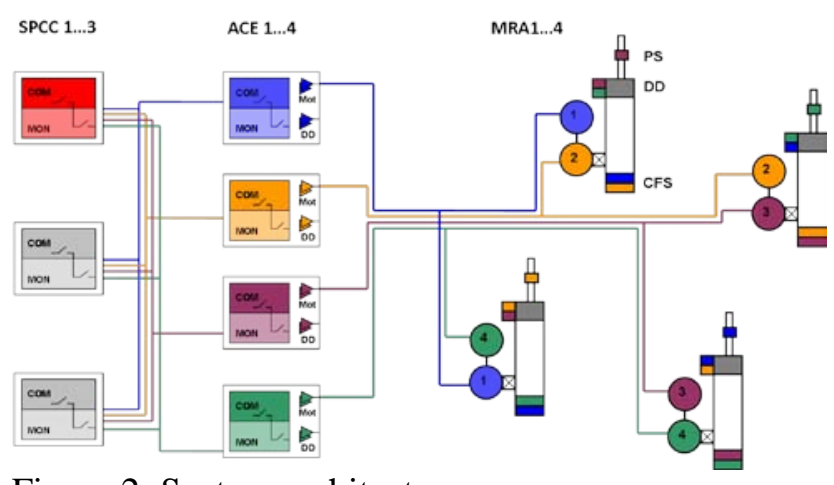

Figure 2: System architecture

Figure 2 shows the applied component mapping. The boxes on the left-hand side represent the topology of the dual-lane computers, namely swashplate control computer (SPCC) and actuator control electronics. Since motor control responsibilities are split and mapped to all four ACEs, they are operating in an active/active configuration. The SPCC functions can be assumed to be functionally integrated in a flight 
control computer (FCC) in a master/slave configuration and therefore its topology will be adopted. Three SPCCs are depicted being the minimum viable degree of redundancy. An ACE additionally contains power stages for motor operation and disconnect device activation, respectively (see triangles in figure 2). In addition to the two motors (circles), each main rotor actuator (MRA) comprises a disconnect device (DD) equipped with dual activation path, two cut force sensors (CFS) and a single position sensor (PS). As can be seen from the respective color coding, the disconnect device is controlled by two ACEs different to those assigned to the two motors of an actuator. This is to allow for disconnection even after both motors were lost due to ACE malfunctions to decouple dead rotary inertia.

\section{Control and monitoring}

In this paragraph the control and monitoring approach is briefly introduced. A more detailed description can be found in [4].

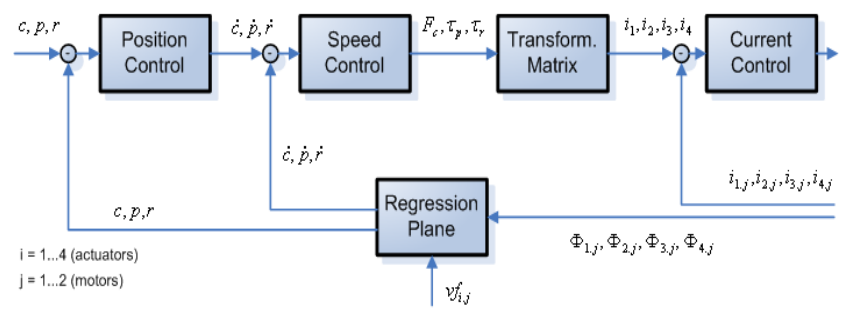

Figure 3: Control architecture

\subsection{Control architecture}

The presented actuator arrangement causes overdetermined kinematics, since four actuators are used to control the three degrees of freedom of the helicopter swashplate (collective, pitch, and roll). A control approach is used which is based on transforming the four actuator position signals into three position parameters, derived from a method introduced by [5]. Control is performed by means of a cascaded PID architecture comprising current, speed and position loop for each of the three directions (figure 3). Eight motor position signals provide position feedback (two resolvers per actuator; the absolute position sensor on actuator level is used for monitoring only). By means of a regression plane the actual swashplate position is determined and transformed into respective actual collective, pitch, and roll values. The three force/torque set values are transformed back into four actuator torque set values, i.e. one per actuator. Hence, each two motors per actuator receive a common torque command. By this set- up force fighting between single actuators is excluded by design for nominal conditions. This approach based on coordinate transformations is a simple and powerful method, which is however threatened if specific failures are not detected.

\subsection{Monitoring architecture}

In order to mitigate the effects of the component failures described above, the system must be fitted with appropriate monitoring. As a general philosophy, simple mechanisms are desired. Therefore, most of the monitoring algorithms rely on redundancy of information and signal comparison. Complex healthmonitoring and the associated knowledge database are avoided. In addition, the control functionality is totally decoupled from fault-detection algorithms for its continuous operation. In other words, control loops are never influenced by ongoing fault detection processes, unless an unambiguous decision was made by the monitoring part.

There are three reconfigurations designed to be automatically executed by the system, namely isolation of faulty position signals, disconnect device activation, and motor shutdown. For this purpose, the monitoring subsystem supervises the sensor signals of all actuators, compares redundant information and generates trusted signals fed to the controllers. Five monitors are continuously assessing parallel tasks:

- Sensor monitor

- Actuator positioning monitor

- Swashplate positioning monitor

- Jam monitor

- Motor monitor

An additional decision layer evaluates the opinions of the independent monitors and initiates the respective reconfiguration. In case a faulty position signal is detected, the respective signal is permanently isolated by excluding it from the regression plane computation by means of a validity factor (see also [4]). The disconnect device is activated via the respective power stages (see figure 2) if a mechanical jam was unambiguously detected. Motor failures are typically detected internally by their dedicated control electronics.

\section{Model implementation}

\subsection{General modeling approach}

For model implementation the Modelica [6] based simulation software Dymola [7] is used. The overall 
system simulation model is shown in figure 4 . Within the blue dotted frame the system components are located (top down order): The lower swashplate (including the inertias of the upper swashplate and the rotor blades) and the respective actuator hinges at the helicopter strucure, the array of actuators, and the control and monitoring blocks. Control (green) and monitoring (orange) loops are depicted. Inputs to the system are aerodynamic forces, power supply and position commands. On the bottom of figure 4 the system parameters are illustrated, assigned to the five categories mechanical drive train (MDT), power stages and motors (PSM), position commands (POS), external forces (FORCE), and failure injection (FAIL).

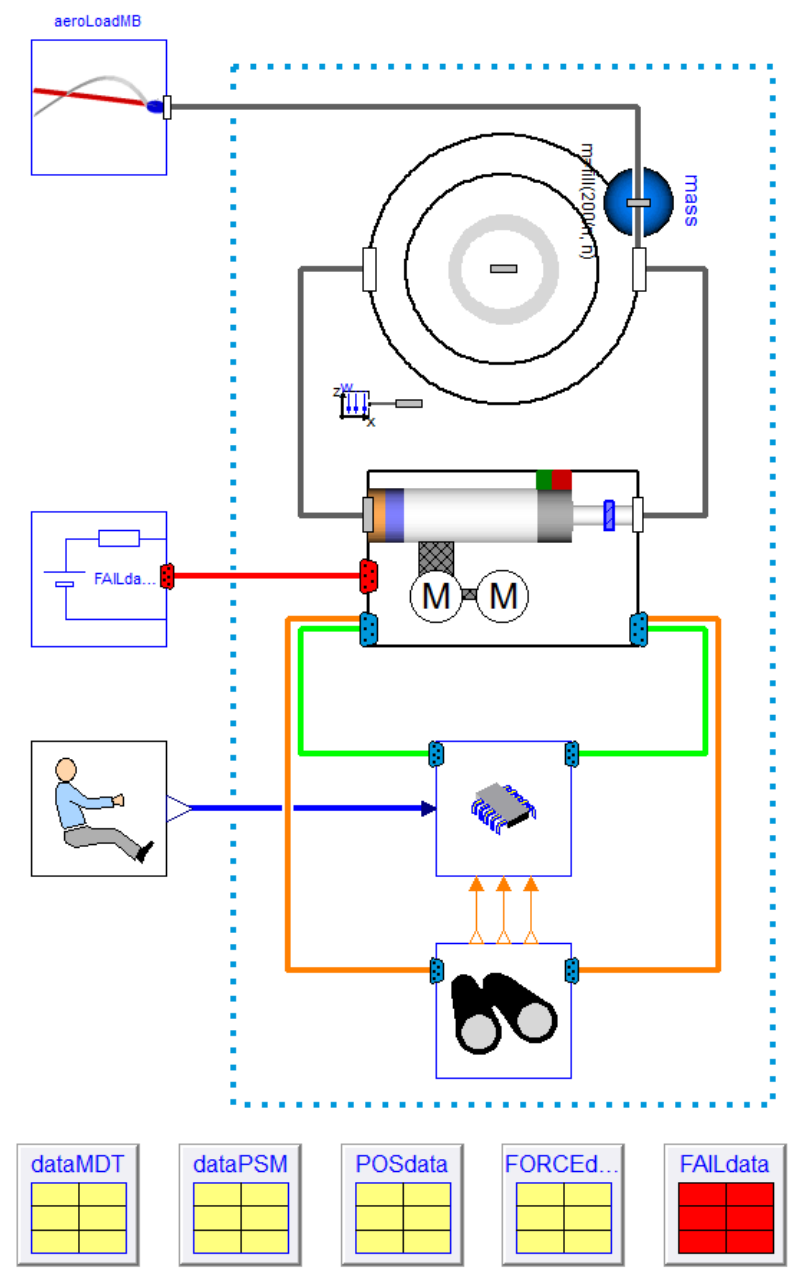

Figure 4: Top layer of system simulation model

A major idea of the simulation model is to investigate not only one specific system design, but to allow for comparison of the performance of several concepts against each other. One important goal of the model therefore is easy generation of models of concept variants. Therefore, for instance the number of actuators is a model parameter in order to allow for variation of the actuator redundancy. The actuators are grouped in an array of components with the respective connectors. Figure 5 illustrates concepts comprising three, four, and five actuators, respectively.

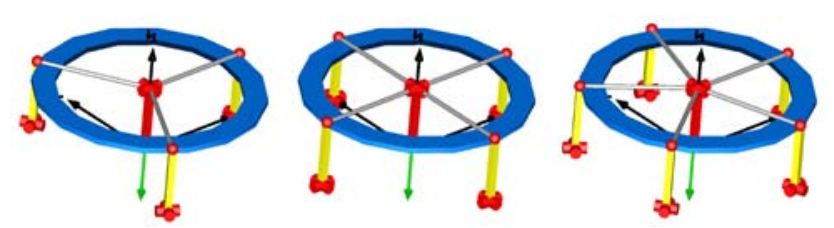

Figure 5: Swashplate actuation design variants

Apart from the Modelica Standard Library no other publically available model library has been used. Class parameterization is applied for the handling of different models of the same component (e.g. drive train with and without friction) and of predefined sets of parameters, e.g. aerodynamic loads, command inputs, and failure cases. Via inheritance fully parameterized simulation experiments were stored, thus facilitating the handling of the large number of simulation test cases to be assessed. The Modelica feature of arrays of components proved to be an essential advantage for the implementation of redundant components. In the following paragraphs the global model components are described.

\subsection{Electromechanical actuator}

As mentioned, an electromechanical actuator consists of a mechanical drive train (including disconnect device), two motors and a power inverter. The disconnect device implementation is based on constraint forces rather than friction: In connected state, internal forces are computed which inhibit relative movement of the disconnect device input and output connections. After activation no more force is transmitted, both parts move independently. The EMA failure cases, namely drive train jamming and drive train fracture, are modeled by activation of a brake and deactivation of a clutch.

For assessment of the effects of mechanical losses in the drive train two implementations have been realized: Friction forces and torques may degrade the overall system dynamics und must therefore be contained in the simulation model. For investigation of the effects of mechanical losses on power consumption an efficiency model has been implemented as an alternative, avoiding the numerical issues and computational load of friction models. 


\subsection{Motor and inverter}

The inverter and motor models are built according to the functional modeling layer specifications [9]. This allows for improvement of the overall system model computational efficiency by exclusion of high frequency switching behavior and reduction of the motor and associated controls model based on the principles described in [8]. Motor controls are implemented using standard space vector control structures with a decoupled control of the current fluxand torque components.

\subsection{Monitoring and control}

The monitoring concept and the control algorithm have been described above. Both are implemented in single model components connected to the array of actuator components. In contrast to the real system implementation the simulation model does not contain redundant computers. The effect of an ACE computer failure can be emulated by switching off the respective power supply. Swashplate control computer (SPCC) topology and failure detection are outside the scope of this paper. The monitoring algorithms are implemented as a sampled block as it would be implemented in flight hardware. Even though an analogue implementation would be preferable for simulation performance reasons, a sampled implementation is required for future hardware-inthe-loop simulations.

\subsection{Aerodynamic forces}

The aerodynamic forces acting on the swashplate and its actuators are given as a sequence of signals assigned to a matrix of flight conditions, e.g. stabilizing, high rate pull up, $30^{\circ}$ turn with severe turbulences, etc.

\subsection{Power supply mapping}

The power supplies are mapped to motors according to the assignment illustrated in figure 2: Each power unit supplies two motors containing to different actuators. Thus the failure of a single power unit does not cause loss of a whole actuator. A dedicated mapping algorithm allows automated mapping of the parameterized power supplies and actuators. It is implemented as variable loops of connect statements.

\subsection{Failure injection}

The simulation model covers a set of relevant failure cases, as they were already introduced above. Table
1 shows a summary of the most relevant component failures and indicated the manner of injection. Each injection is parameterized via setting of a pair of time/ value. Since not all combinations of failures and fail sequences are relevant, predefined sets of parameterizations have been defined as parameter records. All of them are collected in a failure record on the top model hierarchy, while class parameterization allows activation of specific fail cases.

\begin{tabular}{|l|l|l|}
\hline \multirow{2}{*}{ Component } & Failure & Injection \\
\hline \multirow{2}{*}{ Mechanical drive train } & Fracture & Clutch \\
\cline { 2 - 3 } & Jam & Brake \\
\hline \multirow{2}{*}{ Motor-Converter } & No torque & Switch \\
\cline { 2 - 3 } & Short circuit & Switch \\
\hline \multirow{2}{*}{ Power bus } & Power interruption & Switch \\
\cline { 2 - 3 } & Voltage drop & Switch \\
\hline ACE & Shutdown & Power switch \\
\hline \multirow{2}{*}{ Force sensor } & Freeze & Signal Hold \\
\cline { 2 - 3 } & Bias & Signal Add \\
\hline \multirow{2}{*}{ Position sensor } & Freeze & Signal Hold \\
\cline { 2 - 3 } & Bias & Signal Add \\
\hline
\end{tabular}

Table 1: Component failures covered in the current model

\section{Simulation results}

Validation of the system behavior requires a large amount of simulation test cases to be performed. Those are implemented by means of a dedicated test case library which can be re-run on demand. This allows for comparability, consistency and easy reproduction of the total set of test cases. This chapter presents a short selection of simulation results of the most relevant failure cases.

\subsection{Motor failure}

Figure 6a shows the response of the system to a collective position demand signal injected at $t=0.5 \mathrm{~s}$. Both motors of actuator 1 need the same current (fig. 6b) and deliver the same torque (fig. 6c). At 0.6s a winding short circuit failure of motor 1 occurs. Motor 2 now draws more current and delivers nearly double torque, whereas faulty motor 1 shows shortcircuit current but only a small braking torque, while the actuator speed is maintained. At $t=1 \mathrm{~s}$ the position demand is satisfied, the motors continuously reduce speed. Consequently, the speed dependant shortcircuit current of motor 2 almost disappears after 
1.5s. Motor 2 keeps the actuator in steady state. For this simulation the motor monitoring was deactivated in order to check the actuator performance in the case of a motor failure.
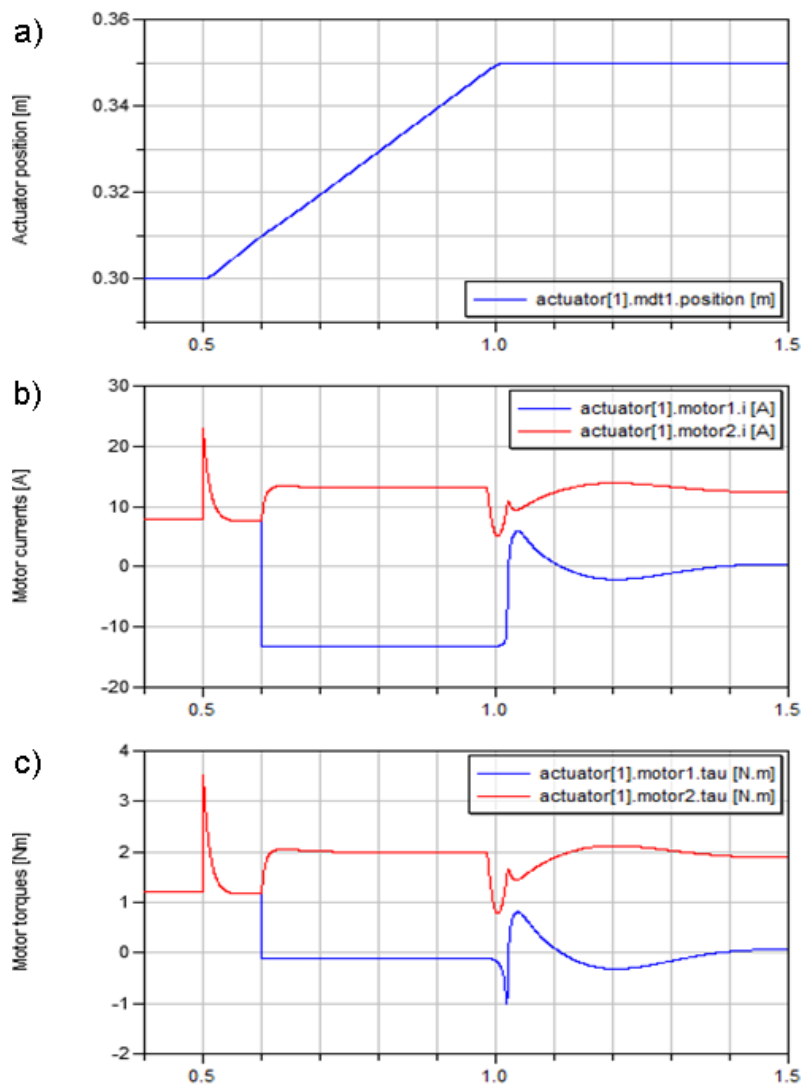

Figure 6: Actuator response on motor short circuit failure

\subsection{Power failure}

Figure 7a shows the response of the system to a collective position demand signal injected at $0.5 \mathrm{~s}$. All power units deliver the nominal voltage of $270 \mathrm{~V}$, all motors draw the same current. At $\mathrm{t}=0.6 \mathrm{~s}$ a first power supply fails. Motor 2 of actuator 1 and motor 1 of actuator 2 subsequently draw no more current (and deliver no torque). Motor 1 of actuator 1 and motor 2 of actuator 2 compensate for this loss, i.e. draw the double current while the actuator speed is maintained.

At $\mathrm{t}=1.1 \mathrm{~s}$ a second power supply fails, thus only 4 out of 8 motors of the overall system remain active. In figure 7c motor 1 of actuator 1 represents the 4 operative motors, the other three of which are not displayed for transparency reasons. The demanded position is maintained, but the system behaves more sensitively. Damping of the current oscillations caused by the second power unit failure requires almost one second. The system remains operational with reduced performance.
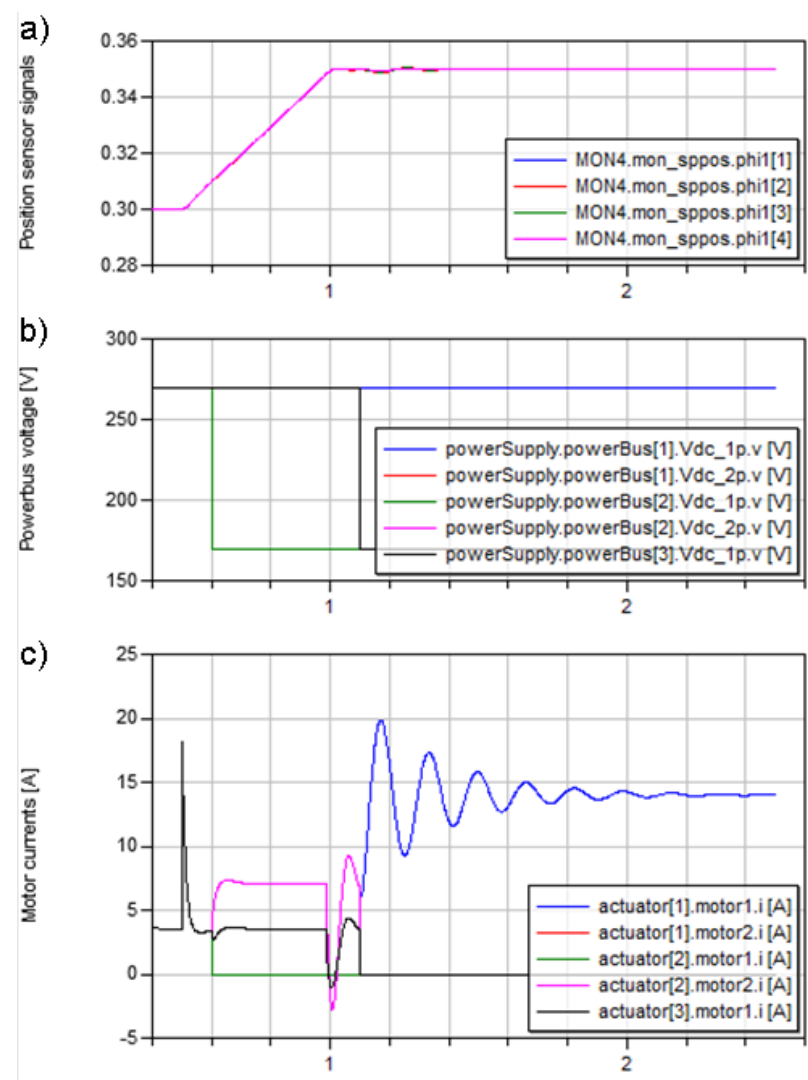

Figure 7: System response on single and double power failure

\subsection{Mechanical jam}

For the jamming scenario the following critical condition is simulated: A sudden friction force causes instantaneous jamming, i.e. unability of displacement. This exposes the system to the most stringent requirement regarding detection time. The disconnect device must be activated rapidly to maintain control stability and limit mechanical stress.

Figure 8 shows the respective simulation results. The swashplate performs a collective movement of $0.05 \mathrm{~m}$ starting at $\mathrm{t}=0.5 \mathrm{~s}$ with maximum speed $\mathrm{v}_{\max }=100 \mathrm{~mm} / \mathrm{s}$. At $\mathrm{t}=0.7 \mathrm{~s}$ a mechanical jam is injected at actuator 1 (fig. 8a, blue lines). Current commands immediately change, expecting mainly actuator 1 to compensate for the position control deviation. This effect highlights the dependency of the controller on sophisticated jam detection: Of all actuators the failed one is powered most, which in turn weakens the remaining operative EMAs. The measured forces however illustrate that actuator 2 and 4 (pink line) sustain almost the full loads, while actuators 1 and 3 do not contribute significantly.

The low measured force at actuator 1 however contradicts to the high commanded current. This effect is used for jam detection by means of an internal torque residual. At $\mathrm{t}=0.9 \mathrm{~s}$ the disconnect device, represented by an idealized mechanical clutch, is acti- 
vated. The converging position signals show that horizontal swashplate attitude is recovered within $0.2 \mathrm{~s}$. Subsequently, for geometrical reasons, actuators 2 and 4 are in charge of sustaining the swashplate loads, while actuator 3 draws current just for stabilization. The motors of the failed actuator are shut down.
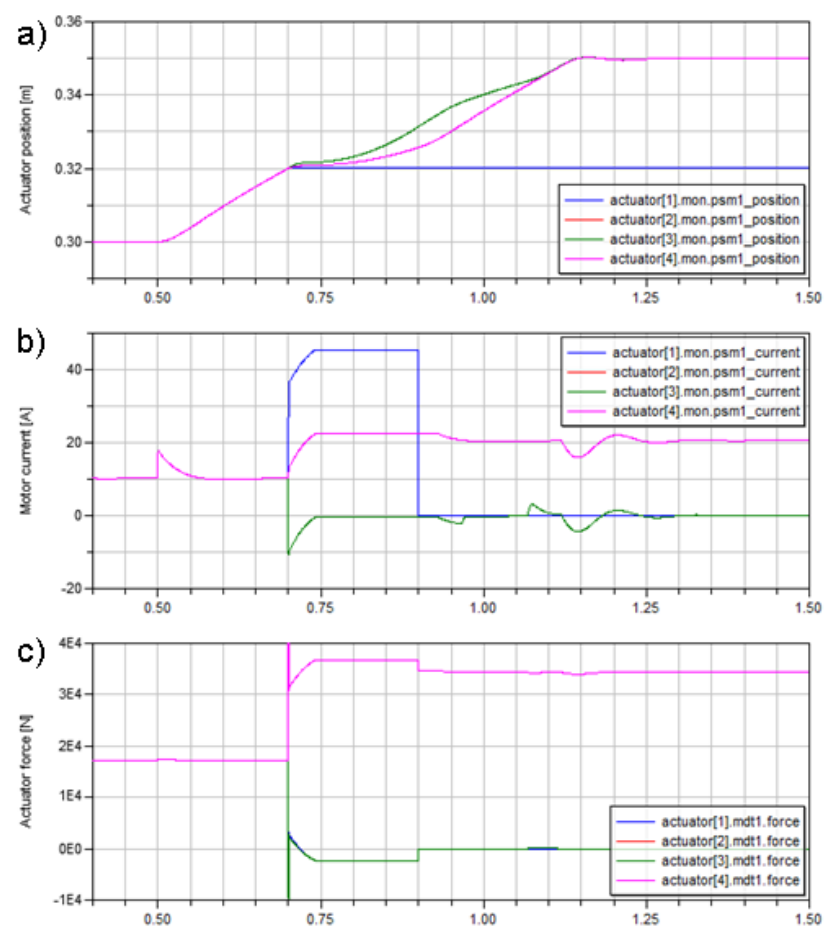

Figure 8: System response on mechanical jam

\subsection{Position signal failure}

Detection and isolation of a failed position sensor is a very important task, since the measured positions are the only signals directly influencing the control loops. Via the regression plane, a non-detected position sensor failure would lead to faulty feedback to the position and speed loop.

Figure 9 depicts signals related to a position sensor failure and its detection. Again, a collective swashplate movement of $0.05 \mathrm{~m}$ is commanded at $\mathrm{t}=0.5 \mathrm{~s}$ with full specified speed. Resolver 1 at actuator 1 fails due to freeze at $\mathrm{t}=0.6 \mathrm{~s}$. This measured value is however be taken into account for the feedback calculation of the swashplate position.

As can be seen from figure 9a, the false position value leads to an increasing diversion of the swashplate from the horizontal plane. In the swashplate positioning monitor the distance of each measured position to the overall reference plane is calculated based on the Hesse normal form. Figure 9b shows that the faulty sensor 1 deviates faster than the others. After exceeding a predefined deviation threshold, this is considered a sensor failure. To avoid that temporary disturbances may lead to a false decision, several confirmation cycles are performed (see figure 9c). At $\mathrm{t}=0.8 \mathrm{~s}$ the decision is confirmed and resolver 1.1 is isolated. As an immediate effect, all remaining position signals perfectly fit to the plane calculated without the failed signal (see figure 9b). Horizontal attitude of the swashplate is recovered and maintained as shown in figure 9 a.
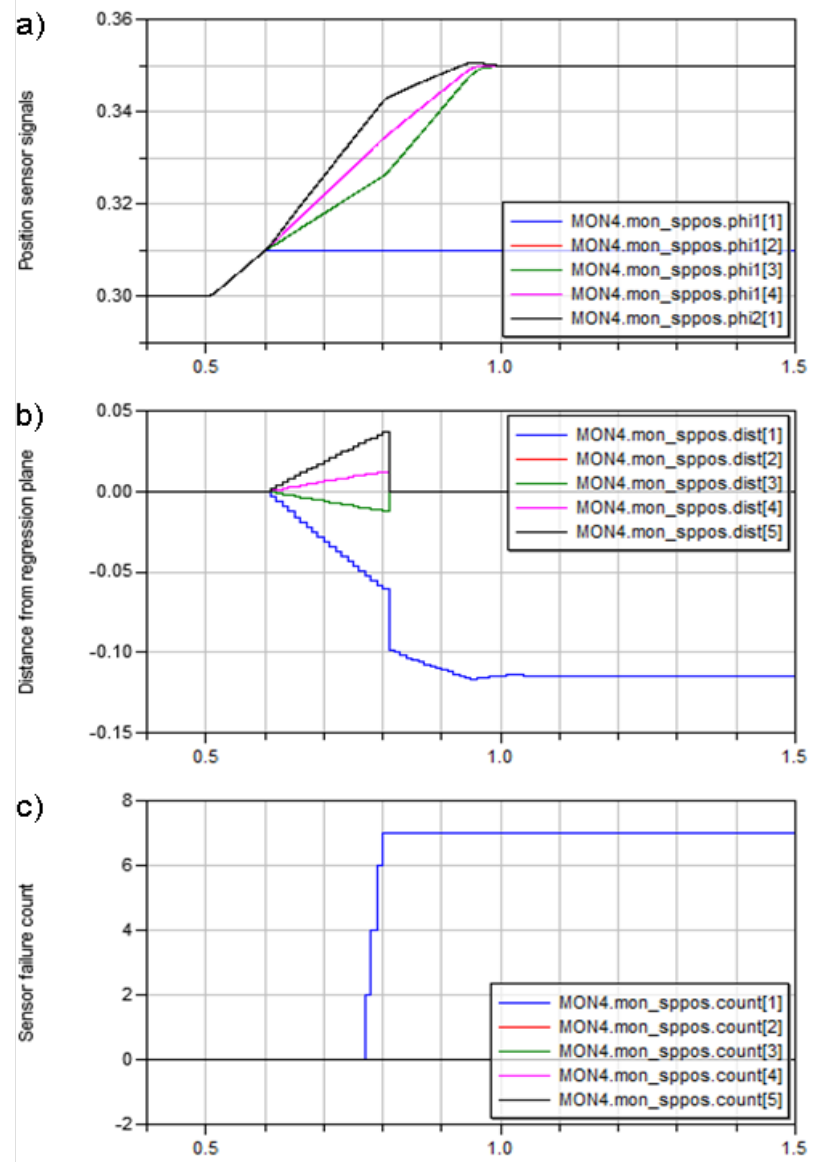

Figure 9: System response on position sensor failure

\section{Conclusion}

The presented paper introduced a safety-critical application of electromechanical actuators. The accompanied challenges of such system were described and a summary of relevant failure cases was given. Modelica is considered a suitable means for modeling of this kind of system including the specific characteristics, such as redundancy, mapping, fault injection, failure detection, and reconfiguration. Exemplary simulation results depicted the system response on specific relevant failure cases. It was shown that performance and reconfiguration behavior are as expected. 


\section{Acknowledgements}

The described investigation is funded by the European Union's Seventh Framework Programme (FP7/2007-2013) for the Clean Sky Joint Technology Initiative under grant CSJU-GAM-SGO-2008-001. The contribution of the motor and inverter models by the project partner University of Nottingham is highly appreciated.

\section{References}

[1] W. Bittner. Flugmechanik der Hubschrauber, Springer Verlag, 2005.

[2] A. Naubert. Pyrotechnic Jam-Relief Mechanism for Electromechanical Actuators in Flight Control Applications, Proceedings of the Actuator12 International Conference, Bremen, Germany, 2012

[3] European Aviation Safety Agency. Certification Specifications for Large Rotorcraft (CS29), Köln, Germany, 2003
[4] S. Seemann, M. Christmann, P. Jänker. Control and Monitoring Concept for a FaultTolerant Electromechanical Actuation System, Proceedings of the R3ASC International Conference, Toulouse, France, 2012

[5] B. K. Walker, E. Gai. A New Approach to Fault-Tolerant Helicopter Swashplate Control, AIAA Aircraft Design, Systems and Technology Meeting, Forth Worth, USA, 1983

[6] www.modelica.org

[7] www.3ds.com/de/products/catia/portfolio/ dymola

[8] T. Wu, S. Bozhko, G. Asher, P. Wheeler. Fast Reduced Functional Model of Electromechanical Actuators for More-Electric Aircraft Power System Study, SAE Technical Paper 2008-01-2859, Nov.2008

[9] S. Bozhko, T. Wu, C.I. Hill, G. Asher. Accelerated simulation of complex aircraft electrical power system under normal and faulty operational scenarios, in Proc. IEEE IECON 2010, Nov.2010, pp 333-338 